Pirineos, 164: 117 a 133, JACA; 2009. ISSN 0373-2568

\title{
EVALUACIÓN DEL CONOCIMIENTO FLORÍSTICO DE LA CORDILLERA CANTÁBRICA (ESPAÑA) A PARTIR DE BASES DE DATOS DE BIODIVERSIDAD
}

\author{
B. JIMÉNEZ-ALFARO
}

\author{
Universidad de Oviedo. INDUROT - Jardín Botánico Atlántico. \\ Avda. del Jardín Botánico, s/n. 33394 Gijón (España)
}

\begin{abstract}
Assessing biodiversity is a pre-requisite for the conservation of any region, and biological databases are an essential tool for estimation of diversity measures. However, biogeographical and administratively fragmented regions are commonly characterized by heterogeneous databases performed by different institutions at different scales. In this study, the floristic knowledge (vascular plants) detected by regional databases in the Cantabrian Range (Spain) is assessed. Georeferenciated and non-overlapping databases provided by a biological collection (herbarium) and a more complete Atlas (herbarium + literature) were compared in terms of historical prospection and spatial patterns (10x10 km UTM grid) of species richness, and their completeness was evaluated using nonparametric estimators (ICE, Chao2, Jack1 and Jack2). Both regional databases were also compared with a national floristic database covering the whole study area. Regional databases detected similar patterns of historical and spatial sampling effort, and observed species richness was highly correlated with the national database, although a high variability in maximum species richness by UTM was detected. Richness estimations showed that regional databases are incomplete, although they permit to estimate the total floristic richness of the Cantabrian Range in c. 3590 species and subspecies. Our results suggest that incomplete, geographically biased and heterogeneous databases offer similar possibilities for detecting under-sampled areas in a given territory, although joining literature with herbarium data may considerably improve species richness estimations. Nevertheless, a complete and cost-effective biodiversity assessment of biogeographical areas characterized by different databases should be only concluded merging all available data. In conclusion, a serious effort to unify regional and other databases in mountain areas is strongly recommended.
\end{abstract}

Keywords: Biodiversity databases, sampling effort, species richness, Cantabrian Range, important plant areas. 
B. JIMÉNEZ-ALFARO

RESUMEN.- La valoración de la biodiversidad es un requisito fundamental para la conservación de cualquier región, y las bases de datos biológicas representan una herramienta básica para la estimación de medidas de diversidad. Sin embargo, muchas regiones biogeográficas fragmentadas administrativamente suelen estar caracterizadas por bases de datos heterogéneas realizadas desde diferentes instituciones y a diferentes escalas. En este trabajo se evalúa el conocimiento florístico de plantas vasculares aportado por bases de datos regionales en la Cordillera Cantábrica (España). Se utilizaron los datos georeferenciados de bases de datos no superpuestas, provenientes de una colección de herbario y de un atlas completado con información bibliográfica, los cuales fueron comparados en función de los patrones temporales y espaciales (cuadrículas UTM de $10 \times 10 \mathrm{~km}$ ) de riqueza florística, utilizando estimadores no paramétricos (ICE, Chao2, Jack1 y Jack2) para medir el grado de conocimiento que las bases de datos reflejan. Ambas fuentes fueron también comparadas con una base de datos nacional con información sobre todo el área de estudio. Las bases de datos regionales detectaron unos patrones similares de prospección histórica y espacial, y los valores de riqueza florística observada estuvieron altamente correlacionados con la base de datos nacional, si bien se detectó una elevada heterogeneidad en la riqueza conocida por UTM. Las estimaciones de riqueza florística indicaron que ambas bases de datos están incompletas, aunque permitieron realizar una estimación de la riqueza total de la Cordillera Cantábrica (en torno a 3590 especies y subespecies). Los resultados obtenidos sugieren que bases de datos incompletas, parciales y de diferente naturaleza pueden aportar información similar para la detección de áreas poco prospectadas, aunque la incorporación de referencias bibliográficas a las bases de datos de herbarios puede ofrecer mejores estimaciones de riqueza florística. Sin embargo, una valoración completa y efectiva en costes de la biodiversidad de zonas biogeográficas caracterizadas por diferentes bases de datos debería sólo concluirse mediante la unión de todos los datos disponibles. En conclusión, se recomienda realizar esfuerzos serios para la unificación de bases de datos regionales y de otro tipo en áreas de montaña.

Palabras clave: bases de datos de biodiversidad, esfuerzo de muestreo, riqueza florística, Cordillera Cantábrica, áreas importantes para las plantas.

\section{Introducción}

La caracterización de la biodiversidad representa un paso necesario para el estudio y conservación de los recursos biológicos de cualquier territorio. Entre los aspectos más estudiados en la valoración biogeográfica de la biodiversidad destacan las medidas de la diversidad alfa, beta o gamma (WHITTAKER, 1972), la relación especies-área (ROSENZWEIG, 2004), o los patrones espaciales que describen la distribución de organismos (NOGUÉS-BRAVO \& MARTINEZ RICA, 2004; GARCÍA \& GÓMEZ, 2007). La medición del número total de especies o el componente endémico también se utiliza como base para la delimitación de áreas de interés (ANDERSON, 2002) o santuarios de conservación como los hotspots (MYERS et al., 2000) y los micro-hotspots 
(MITTERMEIER et al., 1999; MACHADO, 2002). Sin embargo, la caracterización de la biodiversidad de un territorio requiere de listados actualizados sobre el número de taxones y su distribución conocida, así como de una aproximación al grado de fiabilidad de las fuentes de información disponibles. Incluso en áreas teóricamente tan bien prospectadas como Europa, suelen reconocerse importantes lagunas en la disponibilidad de datos sobre biodiversidad (PURVIS \& HECTOR, 2000; KIER et al., 2005), debidas tanto a las limitaciones en el muestreo histórico del territorio como a la existencia de información oculta en colecciones biológicas y en bibliografía poco accesible. Como respuesta a esta situación, en los últimos años se han desarrollado numerosas iniciativas para la recopilación e informatización de datos a partir de colecciones biológicas $\mathrm{u}$ otras fuentes, entre las que destaca la plataforma Global Biodiversity Information Facility (GBIF, 2007). Instituciones como museos de historia natural, jardines botánicos o universidades desarrollan cada vez con más interés bases de datos sobre biodiversidad a escala local, regional o nacional, mientras se proponen sistemas de análisis y valoración de la biodiversidad explotando estas fuentes (PETERSEN et al., 2003; MEIER \& DIKOW, 2004). A pesar de ello, las bases de datos ofrecen aún limitaciones para la estimación de medidas de biodiversidad, debido a que suelen ofrecer información sesgada e incompleta (HORTAL et al., 2007), por lo que se hace necesario evaluar el grado de representatividad que ofrecen, antes de su aplicación para la valoración de cualquier territorio (KIER et al., 2005).

En este trabajo se analiza la información aportada por bases de datos que contienen información parcial sobre la flora vascular de la Cordillera Cantábrica, en el norte de la Península Ibérica. El área de estudio constituye un territorio montañoso dividido en cuatro regiones administrativas diferentes, donde la información disponible sobre diversidad vegetal se gestiona a través de universidades o centros de investigación independientes y de ámbito local. El hecho de que la información existente se acumule de forma heterogénea y en diferentes bases de datos dificulta su análisis sintético, no existiendo aún estudios que traten la flora del territorio desde una perspectiva única. Al igual que ocurre en el conjunto de la Península Ibérica, cuyo inventario se estima aumentará considerablemente en la primera mitad del siglo XXI (CASTROVIEJO, 2002) es posible que el inventario florístico de la flora de la Cordillera Cantábrica esté aún incompleto. Además, y dado que las bases de datos florísticas de la Península Ibérica se desarrollan simultáneamente en el ámbito nacional (ej. ANTHOS, 2007) y local (ej. FONT, 2007), se espera que existan duplicidades de información debidas a la utilización de las mismas fuentes a diferentes escalas. Sin embargo, existen pocos estudios que analicen la relación entre bases de datos heterogéneas que tratan parcialmente un mismo territorio, o su capacidad para describir el conocimiento florístico de 
áreas biogeográficas, como por ejemplo las zonas de montaña. Con el objeto de investigar la capacidad de bases de datos parciales para ofrecer información de utilidad para la valoración de la biodiversidad de la Cordillera Cantábrica, en este trabajo se compara el grado de conocimiento florístico reflejado por las principales bases de datos que afectan al territorio. Los objetivos específicos son: (1) conocer los patrones espaciales y temporales de prospección florística en la Cordillera Cantábrica, detectados por bases de datos basadas en diferentes fuentes de información; (2) analizar el grado de representatividad de riqueza florística que ofrecen bases de datos heterogéneas, utilizando estimadores de riqueza total basados en la intensidad de muestreo.

\section{Material y métodos}

\section{1 Área de estudio y obtención de datos}

La Cordillera Cantábrica representa el extremo occidental del arco alpino europeo (sensu OZENDA, 2002). Se trata de un territorio biogeográfico de transición entre el clima templado-atlántico y mediterráneo, donde son frecuentes las áreas que superan los $2000 \mathrm{~m}$ de altitud (Figura 1). La información de partida utilizada en este estudio proviene de las dos bases de datos florísticas que mayor superficie cubren de la Cordillera Cantábrica: (1) el Atlas de Flora Vascular de Castilla y León (en adelante, BD-CyL), con información referida principalmente a la vertiente meridional, y que contiene información de herbarios, fuentes bibliográficas e informáticas (VVAA, 2007); y (2) el herbario FCO del Departamento de Botánica de la Universidad de Oviedo, Asturias (en adelante, BD-FCO), con información informatizada de todos sus pliegos, procedentes en su mayor parte de la vertiente septentrional. La delimitación de la Cordillera Cantábrica se basó en la unidad orocantábrica reconocida por RIVAS-MARTINEZ et al. (2002), si bien el área de estudio se restringió a 155 cuadrículas UTM de 10x10 km que contenían datos en alguna de las dos bases de datos mencionadas (Figura 1). Ambas fuentes ofrecen información florística sobre flora vascular (espermatófitos y pteridófitos) y pueden considerarse relativamente homogéneas desde un punto de vista taxonómico, dado que provienen de escuelas botánicas afines. La BD-CyL dispone de un mayor número de registros para el área de estudio (58.000) que los disponibles en el herbario FCO (16.000) debido a que contiene registros bibliográficos, además de pliegos de herbario. A efectos comparativos, se recopiló también la riqueza florística disponible para las 155 UTM en el proyecto ANTHOS (2007), cuya base de datos representa la mayor fuente de informa- 
ción florística de la Península Ibérica, procedente de un proyecto que de forma continua e independiente recopila toda la información disponible en publicaciones periódicas, monografías, tesis doctorales, herbarios, etc. Si bien la interpretación taxonómica utilizada en ANTHOS puede presentar divergencias respecto a las bases de datos regionales, se consideró oportuno utilizar esta fuente como elemento comparativo externo, con el objeto de prospectar las divergencias o afinidades ofrecidas en las medidas de riqueza florística conocida. Para la extracción de información de las tres bases de datos (BD-CyL, BD-FCO y ANTHOS) se utilizó información georreferenciada coincidente espacialmente con las 155 cuadrículas UTM de referencia. La información con una precisión mayor (1x1 km o coordenadas GPS) fue referida a la malla de 10x10, mientras que la información no georreferenciada o con escasa precisión no fue utilizada, debido a la dificultad de asignar la localidad original al área de estudio.

\subsection{Análisis de datos}

En las bases de datos regionales (BD-CyL y BD-FCO) se midió la intensidad del muestreo florístico a partir del número de registros producidos en el tiempo, ya fueran pliegos de herbario (utilizando la fecha de recolección) o citas bibliográficas (utilizando la fecha final de publicación), y mediante regresión lineal se midió la relación entre el número de registros y el número de taxones detectados en cada caso. Con el fin de comparar la información aportada por las bases de datos regionales y nacional, se midió la correlación entre el número máximo de especies y subespecies detectado en ambas fuentes para cada cuadricula UTM, utilizando el índice de correlación de Pearson. Finalmente se utilizaron estimadores no paramétricos (COLWELL \& CODDINGTON, 1994) para la riqueza florística potencial en las áreas geográficas cubiertas por las bases de datos regionales. Si bien la aplicación original de estos estimadores se ha enfocado al diseño de muestreos en función de las curvas de acumulación de especies, en los últimos años su uso se ha extendido a la aplicación de estimaciones de riqueza florística o grado de representatividad de bases de datos (SOBERÓN et al., 2000; MEIER \& DIKOW, 2004; PETERSEN et al., 2003, ROSENZWEIG et al., 2003). Para ello debe seleccionarse una unidad de esfuerzo homogénea en el tiempo o en el espacio, en la que se explora cómo el número de especies encontrado se incrementa a medida que aumenta el esfuerzo de muestreo, hasta llegar a un punto en que apenas se incrementa el número de especies (asíntota de una curva). La aplicación de estos estimadores a colecciones biológicas se basa en la definición de series temporales como unidad de esfuerzo (años, lustros, 


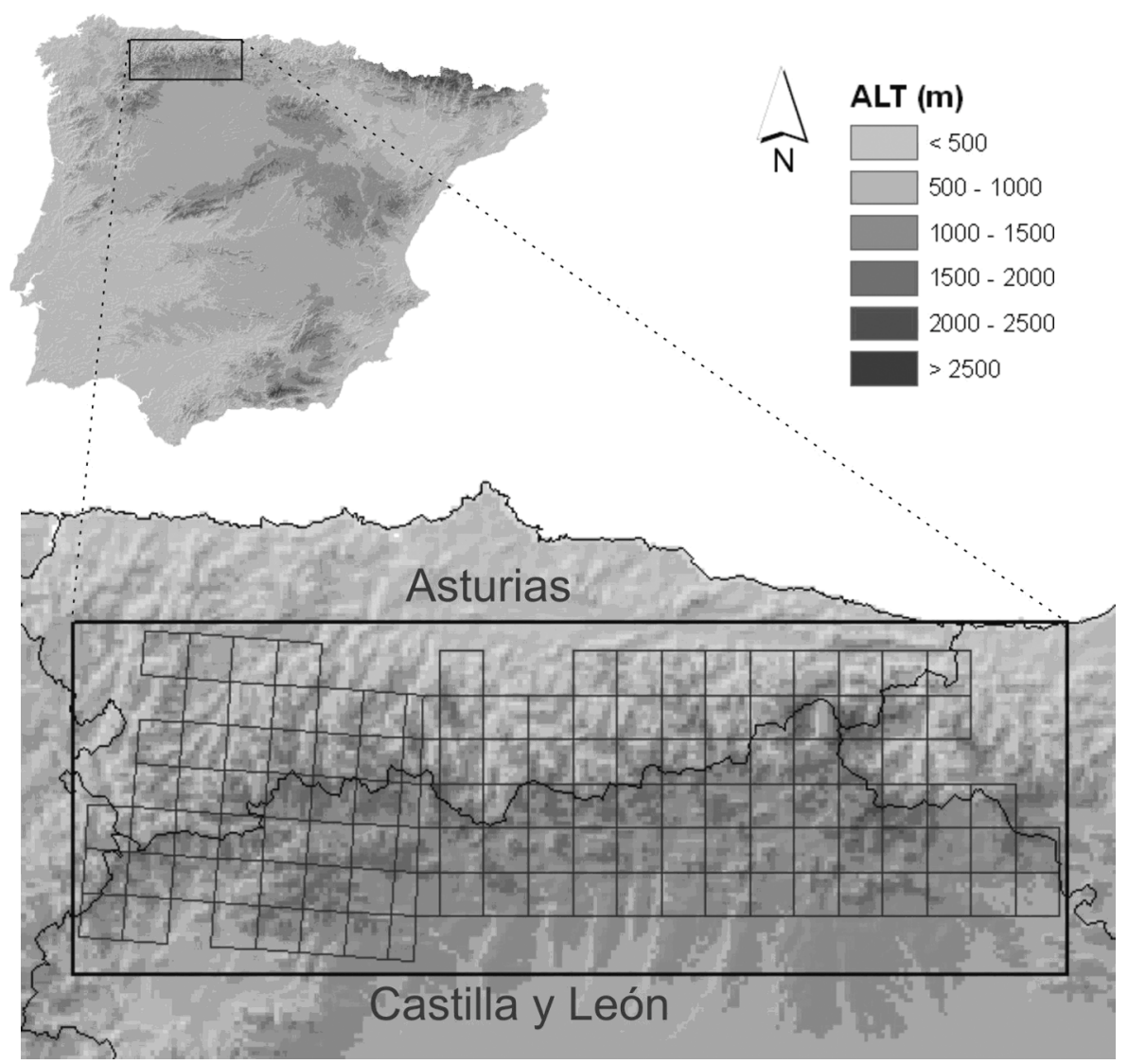

Figura 1. Localización de la Cordillera Cantábrica en la Península Ibérica, y distribución de las 155 cuadrículas UTM de 10x10 km utilizadas como referencia para la extracción de información florística de plantas vasculares en las bases de datos BD-FCO (Asturias) y BD-CyL (Castilla y

$$
\text { León). }
$$

Figure 1. Location of the Cantabrian Range in the Iberian Peninsula and distribution of the 155 UTM grids of $10 \times 10 \mathrm{~km}$ used for obtaining floristic information available in the databases BD-FCO (Asturias) and BD-CyL (Castilla y León).

decenios, etc.; MEIER \& DIKOW, 2002; SOBERÓN et al., 2007). Debido a la posible heterogeneidad de las series temporales, es conveniente excluir las muestras con un número muy bajo de datos, o bien unificarlas de modo que constituyan grupos temporales correlativos (SOBERÓN et al., 2007). A partir de los datos disponibles, se optó por utilizar series temporales anuales, selec- 
cionando sólo aquellos años con un mínimo de 25 plantas muestreadas por año, e integrando los datos de las anualidades con un número inferior con los del periodo siguiente. Finalmente se creó una matriz de presencia/ausencia (filas: taxones; columnas: año o conjunto de años) a la que se aplicaron diferentes algoritmos para la estimación de curvas de acumulación de especies. Se establecieron 300 series aleatorias para la selección del orden de entrada de muestras, aplicando algoritmos diseñados para la estimación de la riqueza total de especies a partir de muestreos incompletos y datos de presencia/ausencia: el estimador ICE (LEE \& CHAO, 1994), basado en los principios de abundancia a partir de la incidencia o porcentaje de casos respecto al total, y que utiliza especies encontradas en diez o menos muestras; Chao 2 (CHAO, 1987), basado en el número de especies raras y en concreto la relación entre las que aparecen en una o dos únicas muestras; Jacknife 1 o de primer orden (HELTSHE \& FORRESTER, 1983) y Jacknife 2 o de segundo orden (SMITH \& VAN BELLE, 1984), basados en los sistemas de reducción de sesgos, y que contabilizan el número de especies que únicamente aparecen en una o dos muestras. La estimación final se calculó a partir del valor medio de cada parámetro, ajustada a un intervalo de confianza del $95 \%$. El tratamiento de los datos se realizó utilizando el programa EstimateS 8.0 (COLWELL, 2005).

\section{Resultados}

\subsection{Prospección florística}

La correlación entre el número de registros (pliegos de herbario, citas bibliográficas o ambos) y el número de taxones por UTM fue elevada, tanto para la BD-CyL $\left(\mathrm{N}=84 ; \mathrm{R}^{2}=0.971 ; \mathrm{p}<0.001\right)$ como para la $\mathrm{BD}-\mathrm{FCO}\left(\mathrm{N}=63 ; \mathrm{R}^{2}=\right.$ 0.962; $\mathrm{p}<0.001)$. En los dos casos los registros disponibles están datados mayoritariamente (>95\%) en la segunda mitad del siglo XX. Los datos referidos al territorio de Castilla y León (BD-CyL) muestran una mayor intensidad de muestreo durante las décadas de los años ochenta y noventa, mientras que en el caso de los datos de herbario disponibles en el herbario FCO, el máximo de registros se corresponde con la década de la década de los 70 y 80 .

La correlación entre el número de especies y subespecies asignado por las bases de datos regionales y ANTHOS fue significativa, y mayor en la base de datos de Castilla y León ( $\mathrm{N}=84$; Coef. Pearson $=0.807$; $\mathrm{p}<0.001$ ) que en el herbario FCO $(\mathrm{N}=63$; Coef. Pearson $=0.557 ; \mathrm{p}<0.001)$. Las fuentes regionales ofrecieron niveles más bajos de riqueza florística en 92 de las 155 UTM (59\%), 
incluyendo 30 cuadriculas en las que únicamente existían registros de ANTHOS. En las UTM con datos compartidos por ambas fuentes (Figura 2) el balance fue igualmente positivo para la base de datos ANTHOS, aunque más equilibrado en el caso de la $\mathrm{BD}-\mathrm{CyL}$ ( $51 \%$ de UTM con menor riqueza florística en la fuente regional) que en el caso de la BD-FCO (57 \%). Las diferencias totales entre cuadriculas fueron muy variables, con un valor máximo en los bordes geográficos de cada territorio (Figura 2) que se atribuyen a la disparidad espacial entre los límites administrativos y las cuadrículas UTM.
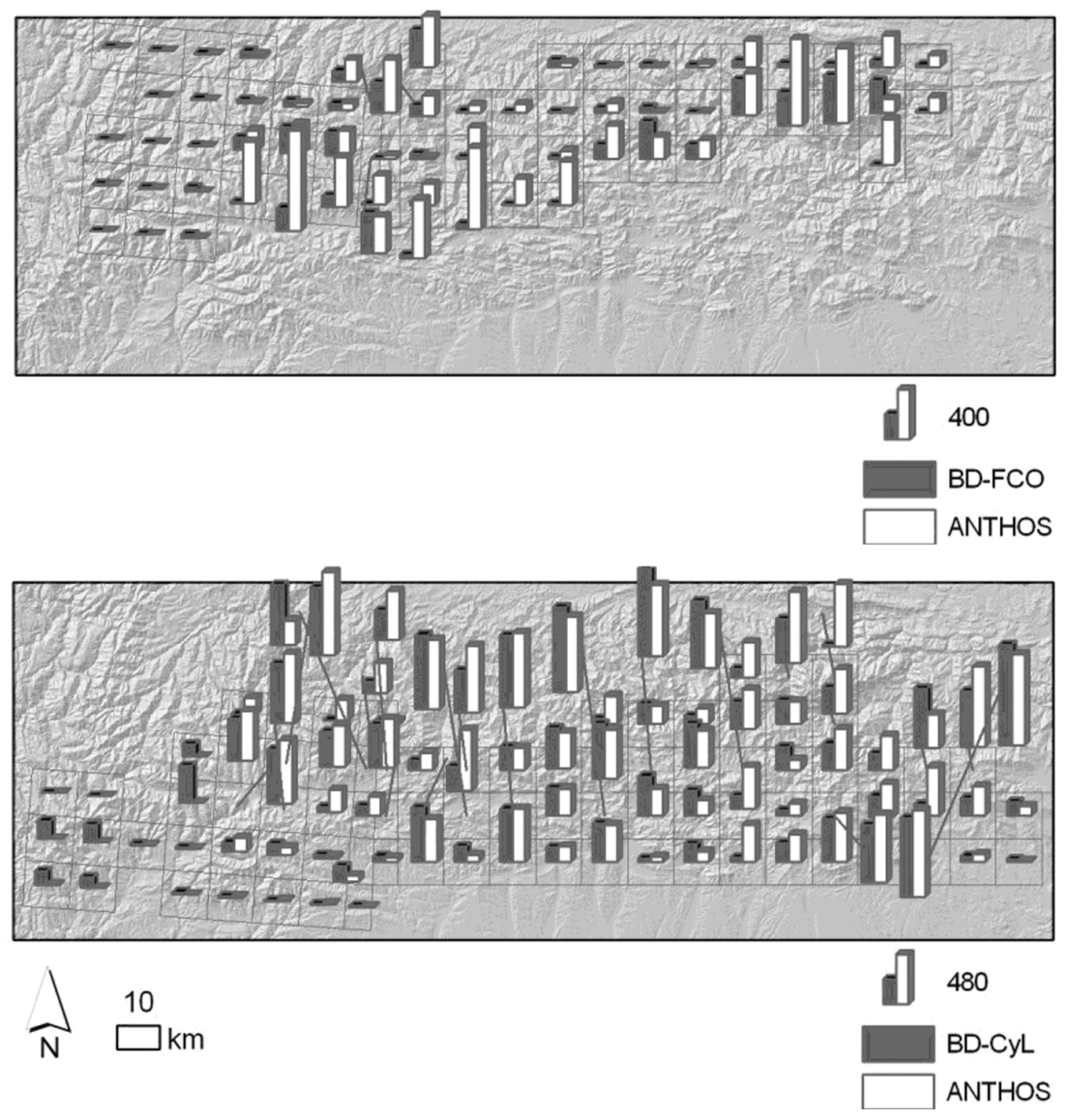

Figura 2. Comparación entre la riqueza florística documentada por la base de datos nacional (ANTHOS) y las bases de datos regionales disponibles para Asturias (BD-FCO) y Castilla y León (BD-CyL).

Figure 2. Comparisons between the floristic richness documented by Spanish national database (ANTHOS) and the regional databases from Asturias (BD-FCO) and Castilla y León (BD-CyL). 


\subsection{Riqueza florística}

El número máximo de taxones registrados para cada UTM ofreció un valor medio de riqueza florística $=224$ (desv. est. $=226 ; \mathrm{N}=155)$ (Figura 2). Las estimaciones realizadas por los diferentes algoritmos para los dos territorios cubiertos por las bases de datos regionales fueron muy similares (Figura 3), si bien en ambos casos el estimador Jack2 ofreció valores ligeramente superiores al resto (Tabla 1). En el caso del ámbito orocantábrico de Castilla y León (BDCyL) el valor medio estimado fue de 2900 especies y subespecies, frente a las 2495 reflejadas por los registros disponibles. En Asturias (BD-FCO) la media estimada alcanzó los 2471 taxones, frente a las 1632 plantas actualmente reconocidas. Se calculó la media de todas las estimaciones (ICE, Chao2, Jack1 y Jack2) como valor estimado medio de riqueza florística (Nest), calculando un índice $C$ para definir el grado de conocimiento del territorio (SOBERÓN et al., 2007) como resultado del cociente especies observadas / especies estimadas $\left(S_{o b s} / S_{e s t}\right)$. El parámetro $C$ puede evidenciar el grado de conocimiento general del territorio, a partir de las muestras incluidas en las bases de datos. En función de ello, el Atlas de Castilla y León reflejó con una mayor exactitud

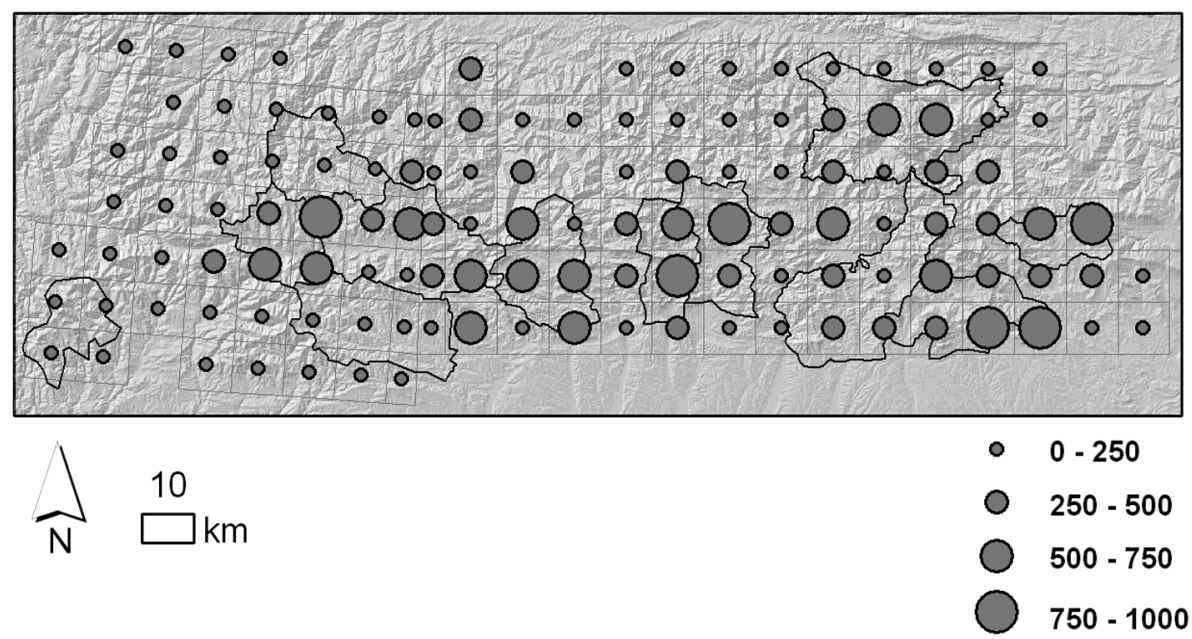

Figura 3. Máxima riqueza florística para cada UTM, en función del valor máximo ofrecido por las bases de datos regionales y nacional. Los polígonos indican las áreas geográficas estudiadas por tesis doctorales y otros estudios florísticos intensivos.

Figure 3. Maximum floristic richness for each UTM, as indicated by national and regional databases (maximum value given by any database was selected). Polygons indicate the geographical areas studied by doctoral thesis and other systematic floristic studies. 
$(\mathrm{C}=0.83)$ la biodiversidad del territorio cubierto, mientras que la base de datos del herbario FCO ofreció una menor representatividad $(C=0.66)$. Considerando como nivel de conocimiento medio del área de estudio el promedio de ambos valores $(C=0.74)$, y aplicándolo al número de taxones definidos en la BD ANTHOS ( $\mathrm{N}=2850)$, se estimó el total de la flora vascular del territorio en $3590( \pm 162)$ taxones.

\section{Discusión}

Al igual que muchos otros territorios, la Cordillera Cantábrica puede considerarse un área florísticamente prospectada de forma desigual. Desde un punto de vista espacial, la variabilidad en el número de registros reconocidos en las 155 cuadriculas UTM indica un muestreo heterogéneo, que se puede relacionar fácilmente con el desarrollo de trabajos de prospección intensivos, como tesis doctorales de tipo florístico (Figura 3). Los bajos valores de riqueza descritos por las bases de datos en algunas UTM permiten reconocer áreas muy poco prospectadas en la zona occidental y la parte central de la vertiente septentrional (Figuras 2 y 3 ), en donde deberían centralizarse futuras campañas de prospección botánica. Desde el punto de vista histórico, las dos bases de datos analizadas señalan la segunda mitad del siglo XX, y de modo especial las décadas de 1970 y 1980, como el periodo de mayor prospección botánica, lo que concuerda con la tendencia general indicada para otros territorios ibéricos (GARCÍA \& GÓMEZ, 2007). En el caso de la Cordillera Cantábrica, la prospección histórica tardía parece más evidente, considerando el elevado número de registros disponibles para la segunda mitad del siglo XX (> 95\% del total). Dicho periodo se ha reconocido como el de mayor descripción de plantas endémicas, sujeto a una prospección florística posterior al de regiones próximas, como las montañas pirenaicas (JIMENEZ-ALFARO et al., 2009).

Las estimaciones de riqueza florística (Tabla 1 y Figura 4) indican que las dos bases de datos analizadas pueden considerarse incompletas, en el sentido de que no reflejan eficazmente la riqueza florística total presente en el área de estudio. La base de datos de Castilla y León (BD-CyL) ofreció un grado de conocimiento $(C)$ más completo, lo que se explica por el mayor número de registros que contiene, al incorporar referencias bibliográficas. Dado que muchos de los registros bibliográficos incluidos en la BD-CyL corresponden a referencias indirectas a pliegos de herbario, resulta difícil evaluar la aportación real de las citas bibliográficas frente a los datos contenidos en los herbarios, en términos de riqueza florística. Sin embargo, las diferencias detectadas en las dos bases de datos regionales sugieren que el papel de los herbarios 
como bases de datos para la evaluación de la biodiversidad mejora sustancialmente cuando se incorporan referencias complementarias, al menos para la aplicación de estimaciones de riqueza basadas en el esfuerzo de muestreo. En este sentido, la forma adoptada por las curvas de acumulación obtenidas a partir de la BD-CyL, con una menor pendiente en su tramo final (Figura 3), indica una cierta saturación de muestras, y por lo tanto una mayor representatividad de la diversidad total, coherente con el elevado valor de $C(=0.83)$ obtenido. Aunque la similitud de las cuatro estimaciones otorga una mayor fiabilidad a los resultados, su interpretación debe considerarse en todo momento como una aproximación a la riqueza florística del área de estudio, teniendo en cuenta las limitaciones estadísticas de estos métodos cuando son utilizados en colecciones biológicas (PETERSEN et al., 2003).

Tabla 1. Medidas de riqueza florística obtenidas a partir de las bases de datos regionales (BDFCO y BD-CyL) para el área de estudio, en función de los estimadores ICE, Chao2, Jack1 y Jack2. Nobs: Número total de especies registradas. Nest: Número medio estimado ( \pm desviación estándar). C: grado de conocimiento.

Table 1. Floristic richness measures obtained from the regional databases regionales (BD-FCO and BD$\mathrm{CyL})$ in the study area, using the nonparametric estimators ICE, Chao2, Jack1 y Jack2. Nobs: Total number of documented species. Nest: Estimated mean ( \pm standard deviation). C: completeness.

\begin{tabular}{|lcc|}
\hline & BD-FCO & BD-CyL \\
\hline Nobs & 1632 & 2435 \\
\hline ICE & 2424 & 2750 \\
Chao2 & 2405 & 2819 \\
Jack1 & 2336 & 2925 \\
Jack2 & 2719 & 3107 \\
\hline Nest & $2471( \pm 147)$ & $2900( \pm 135)$ \\
\hline C (Nobs / Nest) & 0.66 & 0.83 \\
\hline
\end{tabular}

Aun considerando la limitación de utilizar las bases de datos regionales por separado, y sin disponer de una recopilación taxonómica única para el área de estudio, resulta sorprendente el elevado número total de taxones estimados para cada área, así como la riqueza florística estimada en el conjunto del territorio (en torno a las 3590 especies y subespecies). Si bien serán necesarios estudios más completos para obtener una estimación más fiable, los resultados obtenidos indican que el $26 \%$ de la riqueza florística de la Cordillera Cantábrica podría no estar aún documentada en las bases de datos disponibles, aunque es probable que dicho valor esté magnificado por la duplicación de taxones no incorporados en ninguna de las bases de datos 

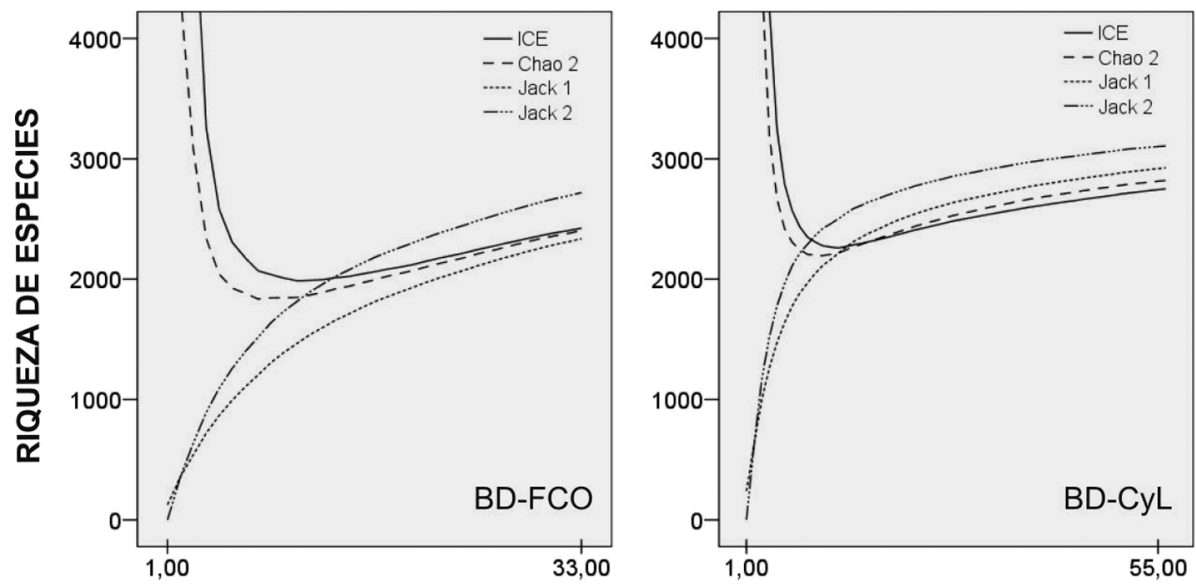

SERIES TEMPORALES

Figura 4. Curvas de acumulación utilizadas para las estimaciones no paramétricas de la riqueza florística a partir de las bases de datos regionales (BD-FCO y BD-CyL), para cada uno de los cuatro algoritmos utilizados (ver Tabla 1 ).

Figure 4. Species curves used for nonparametric estimations of floristic richness in the regional databases Asturias (BD-FCO and BD-CyL), for the 4 algorithmes used (see Table 1).

regionales. En cualquier caso, utilizando un rango posible de entre 3000 y 3500 especies y subespecies, la riqueza florística de la Cordillera Cantábrica contendría cerca del $40 \%$ de la flora ibérica, estimada entre 7500 y 8000 taxones (CASTROVIEJO, 2002). Estos valores resultan especialmente altos en comparación con la riqueza florística de las zonas de mayor biodiversidad de Europa (Tabla 2), lo que podría explicarse a partir de la especial ubicación biogeografía de la Cordillera Cantábrica, con un marcado carácter ibérico y por lo tanto refugio florístico pleistocénico (AKEROYD \& HEYWOOD, 1994), pero con una influencia de ámbitos tan dispares como el mundo atlántico, mediterráneo y boreo-alpino. Los datos ofrecidos por las bases de datos consultadas sugieren que, en función de la riqueza florística y el porcentaje de especies endémicas conocida, la Cordillera Cantábrica deba reconocerse como un área importante para las plantas (ANDERSON, 2002) en Europa occidental, de cara a las estrategias de conservación vegetal (WILLIAMS et al., 2003). 
Tabla 2. Riqueza florística (RF), número de especies endémicas (Nend) y porcentaje de endemicidad $(\%)$ en diferentes regiones montañosas de Europa Occidental.

Table 2. Floristic (RF), number of endemic species (Nend) and percentage of endemicity (\%) in different mountains regions of Western Europe.

\begin{tabular}{lcccl}
\hline Área & $\boldsymbol{R F}$ & Nend & $\%$ & Referencias \\
\hline Pirineos & $3480^{1}$ & 174 & 5 & VILLAR \& GARCÍA, 1989 \\
Sistema Central & - & 65 & - & RIVAS MARTÍNEZ et al., 1986 \\
Cordillera Cantábrica & $2850^{1} / 3590^{2}\left(^{*}\right)$ & 74 & $2,0 / 2,6$ & JIMENEZ-ALFARO et al., 2009 \\
Sierra Nevada & $2100^{1}$ & 80 & 3,8 & BLANCA, 2001 \\
Sur Península Ibérica & $3680^{2}$ & 553 & 15,2 & MELENDO et al., 2003 \\
Sureste de Francia & $3000^{2}$ & 215 & 7,2 & MÉDAIL \& VERLAQUE, 1997 \\
Alpes marítimos & $3605^{2}$ & 115 & 3,2 & CASAZZA et al., 2005 \\
\hline
\end{tabular}

${ }^{1}$ conocida (known); ${ }^{2}$ estimada (estimated); $\left({ }^{*}\right)$ presente estudio (this study).

A pesar de las diferencias cualitativas y cuantitativas en los registros disponibles, las bases de datos regionales (BD-CyL y BD-FCO) coincidieron en señalar las zonas menos prospectadas y la tendencia del muestreo histórico en la Cordillera Cantábrica, a partir de los territorios donde disponen de información. Si bien la riqueza florística reflejada por la base de datos ANTHOS fue en línea general mayor que la de las bases de datos regionales, la alta variación de los valores máximos (Figura 2) y el elevado número de cuadrículas UTM con escasa prospección y por lo tanto baja riqueza florística detectada (Figura 3), indican un muestreo sesgado del territorio en las tres fuentes de datos, a la escala de referencia $(10 \times 10 \mathrm{~km})$. Aunque la correlación detectada entre las bases regionales y nacional confirma la existencia de fuentes comunes (explicada a partir de una misma información disponible en herbarios y/o bibliografía botánica) las diferencias detectadas indican una desigual disponibilidad de información o, cuando menos, una desigual informatización de los datos. Las tres bases de datos analizadas parecen haber duplicado esfuerzos para la informatización de unas fuentes comunes, haciéndolo además de forma desigual, dada la heterogeneidad de información recogida para cada UTM. Dado que, en términos relativos, la proporción de taxones inventariados para cada UTM no varía en función de la fuente consultada, no es posible valorar si las bases de datos regionales o bien la nacional reflejan mejor el conocimiento florístico del área de estudio. Aunque puede concluirse que cualquiera de las fuentes consultadas puede aportar cierta información de interés para la valoración de la diversidad vegetal en el territorio, las limitaciones de los datos disponibles en cada una de las bases de datos por separado siguen siendo relevantes. Teniendo en cuenta la baja efectividad y el sobrecoste derivado de duplicar esfuerzos de informatización en un mismo 
territorio, sería recomendable la unificación de todos los datos disponibles, utilizando criterios taxonómicos consensuados y sistemas informáticos comunes (en este caso, creando una base de datos cantábrica o bien unificando la base de datos nacional), sin olvidar el diseño de nuevos muestreos en las áreas poco prospectadas. En este sentido, este estudio ha demostrado la necesidad de que las regiones biogeográficas gestionadas por distintas administraciones o centros de investigación unifiquen sus bases de datos (KORNER et al., 2007) para obtener estimas fiables de riqueza florística y, en último caso, una acertada valoración de la biodiversidad.

\section{Agradecimientos}

La recopilación de información florística de la Cordillera Cantábrica fue posible gracias a la colaboración de la Junta de Castilla y León (Consejería de Medio Ambiente, Dirección general del Medio Natural) y de la conservadora del herbario FCO de la Universidad de Oviedo (M. Carmen Fernández Carbajal), quienes permitieron el acceso a la información contenida en sus bases de datos. Jorge Soberón aportó valiosas sugerencias y material inédito para la aplicación de los estimadores de riqueza florística, mientras que Álvaro Bueno y José Antonio Fernández Prieto hicieron interesantes aportaciones al estudio.

\section{Referencias}

AKEROYD, J. R. \& HEYWOOD, V. H. (1994). Regional overview: Europe. In: Davis, S. D., Heywood, V. H. \& Hamilton, A. C. (eds.), Centres of Plant Diversity. Volume 1. Europe, Africa, Southwest Asia and the Middle East. WWF \& IUCN, pp. 39-75.

ANDERSON, S. (2002). Identificación de áreas importantes para las plantas (Important Plant Areas - IPA's). Resumen del manual de selección de los sitios para Europa. Plant Life Internacional.

ANTHOS (2007). Sistema de información de las plantas de España. Real Jardín Botánico, CSIC - Fundación Biodiversidad. Recurso electrónico en www.anthos.es. Consulta realizada en diciembre de 2007.

BLANCA, G. (y colaboradores) (2001). Flora amenazada y endémica de Sierra Nevada. Junta de Andalucía/Universidad de Granada, Granada. 410 pp.

CASAZZA, G.; BARBERIS, G. \& MINUTO, L. (2005). Ecological characteristics and rarity of endemic plants of the Italian Maritime Alps. Biological Conservation 123: 361-371. 
CASTROVIEJO, S. (2002). Riqueza florística de la Península Ibérica e Islas Baleares. El proyecto "Flora ibérica". En: PINEDA, F.D.; DE MIGUEL, J.M.; CASADO, M.A. \& MONTALVO, J. Eds. La diversidad biológica de España. Pearson Educación. Madrid.

CHAO, A. (1987). Estimating the population size for capture-recapture data with unequal catchability. Biometrics 43: 783-791.

COLWELL, R.K. (2005). EstimateS: Statistical estimation of species richness and shared species from samples. Version 8.0. User's Guide and application. Published at: http:// purl.oclc.org/ estimates.

COLWELL, R.K. \& CODDINGTON, J.A. (1994). Estimating terrestrial biodiversity through extrapolation. Philosophical Transactions of the Royal Society (Series B), 345: 101-118.

FONT, X. (2007). Mòdul Flora i Vegetació. Banc de Dades de Biodiversitat de Catalunya. Generalitat de Catalunya i Universitat de Barcelona. (año de consulta, 2007). http:/ / biodiver.bio.ub.es/biocat/homepage.html.

GARCIA, M.B. \& GOMEZ, D. (2007). Flora del Pirineo Aragones. Patrones espaciales de biodiversidad y su relevencia para la conservacion. Pirineos 162: 71-88.

GBIF (2007). Informe preliminar del estado de las colecciones de historia natural en España. $\quad$ www.gbif.es/ficheros/GBIF.ES_Inf_Col.pdf GBIF (2007). Infraestructura mundial de información en biodiversidad en España. www.gbif.es/index.php

HELTSHE, J. \& FORRESTER, N.E. (1983). Estimating species richness using the jackknife procedure. Biometrics 39: 1-11.

HORTAL, J.; LOBO, J.M. \& JIMÉNEZ-VALVERDE, A. (2007). Limitations of Biodiversity Databases: Case Study on Seed-Plant Diversity in Tenerife, Canary Islands Conservation Biology 21(3): 853-863.

JIMENEZ-ALFARO, B.; BUENO, A. \& FERNANDEZ PRIETO J.A. (2009). Flora vascular endémica y subendémica orocantabrica. En: LLAMAS, F. \& C. ACEDO. Eds. Botánica Pirenaico-Cantábrica en el siglo XXI. Área de Publicaciones de la Universidad de León, León. Pp. 145-164.

KIER, G.; MUTKE1, J.; DINERSTEIN E.; RICKETTS, T.H.; KÜPER, W.; KREF, H. \& BARTHLOTT, W. (2005). Global patterns of plant diversity and floristic knowledge. Journal of Biogeography 32: 1107-1116.

KÖRNER, H.; DONOGHUE, M.; FABBRO, T.; HÄUSER, CH.; NOGUÉSBRAVO, D.; KALIN ARROYO, M. T.; SOBERON, J.; SPEERS, L.; SPEHN, E. M.;. SUN, H; TRIBSCH, A.; TYKARSKI, P \& ZBINDEN N. (2007). Creative Use of Mountain Biodiversity Databases: The Kazbegi Research Agenda of GMBA-DIVERSITAS. Mountain Research and Development 27(3): 276-281. 
LEE, S.-M., \& A. CHAO. (1994). Estimating population size via sample coverage for closed capture-recapture models. Biometrics 50: 88-97.

MACHADO, A. (2002). La biodiversidad de las islas Canarias. En: PINEDA, F.D. MIGUEL, J.D., CASADO, M.A. \& MONTALVO, J. Eds. La diversidad biológica de España. Pearson Educación. Madrid.

MÉDAIL, F. \& VERLAQUE, R. (1997). Ecological characteristics and rarity of endemic plants from southeast France and Corsica: implications for biodiversity conservation. Biological Conservation 80: 269-281.

MEIER, R. \& DIKOW, T. (2004). Significance of Specimen Databases from Taxonomic Revisions for estimating and mapping the global species diversity of invertebrates and repatriating reliable specimen data. Conservation Biology 18 (2): 478-488.

MELENDO, M.; JIMÉNEZ, E.; CANO, E.; GÓMEZ-MERCADO, F. \& VALLE, F. (2003). The endemic flora in the south of the Iberian Peninsula: Taxonomic composition, biological spectrum, pollination, reproductive mode and dispersal. Flora 198: 260-276.

MITTERMEIER, R.A.; MYERS, N.; ROBLES-GIL, P. \& MITTERMEIER, C.G. Eds. (1999). Hotspots. Earth's Biologically Richest and Most Endangered Terrestrial Ecoregions. CEMEX/Agrupación Sierra Madre, Mexico City.

MYERS, N.; MITTERMEIER, R.A.; MITTERMEIER, C.G.; DA FONSECA \& KENT, J. (2000). Biodiversity hotspots for conservation priorities. Nature 40(3): 853-858.

NOGUÉS-BRAVO D. \& MARTINEZ RICA J.P. (2004). Patrones de distribución espacial de la riqueza de especies de vertebrados en un área de transición biogeográfica: de la Navarra pirenaica a la mediterránea. Pirineos 158-159: 87-106.

OZENDA, P. (2002). Perspectives pour une geobiologie des montagnes. Presses Polytechniques et Universitaires Romandes. Lausanne. 195 pp.

PETERSEN, F.T.; MEIER, R \& LARSEN, M.N. (2003). Testing species richness estimation methods using museum label data on the Danish Asilidae. Biodiversity \& Conservation 12: 687-701.

PURVIS, A. \& HECTOR, A. (2000) Getting the measure of biodiversity. Nature 405: 212-219.

RIVAS MARTÍNEZ, S.; FERNÁNDEZ GONZÁLEZ, F. \& SÁNCHEZ-MATA, D. (1986). Endemic taxa of the Iberian Central System: distribution and ecology. En: Plant Conservation in Botanic Gardens. Ed. J. Cramer.

RIVAS-MARTÍNEZ, S.; DÍAZ GONZÁLEZ, T.E.; FERNÁNDEZ GONZÁLEZ, F.; LOIDI, J.; LOUSA, P. \& PENAS, A. (2002). Vascular plant communities of Spain and Portugal. Itinera Geobotánica 15.

ROSENZWEIG, M.L. (2004). Applying species-area relationships to the conservation of species diversity. En: LOMOLINO, M.V. \& HEANEY, L.R. 
Eds. Frontiers of biogeography: New Directions in the Geography of Nature. Sinauer Assoc., Sunderland.

ROSENZWEIG, M.L.; TURNER, W.R.; COX, J.C \& RICKETTS, T.H. (2003). Estimating Diversity in Unsampled Habitats of a Biogeographical Province. Conservation Biology 17(3): 864-874.

SMITH, E.P. \& VAN BELLE, G. (1984). Nonparametric estimation of species richness. Biometrics 40: 119-129.

SOBERÓN, J.; LLORENTE, J.B. \& OÑATE, L. (2000). The use of specimen label databases for conservation purposes: an example using Mexican Papilionid and Pierid butterflies. Biodiversity \& Conservation 9(10): 14411466.

SOBERÓN, J.; JIMÉNEZ, R.; GOLUBOV, J. \& KOLEFF, P. (2007). Assessing completeness of biodiversity databases at different spatial scales. Ecography 30(1): 152-160.

VILLAR, L., \& GARCIA, B. (1989). Verse une banque de données des plantes vasculaires endémiques des Pyrénées. Acta Biologica Montana 9: 261-274.

VV.AA. (2007). Base de datos del Catálogo de Flora Vascular Silvestre de Castilla y Leon. Universidad de Salamanca, Junta de Castilla y Leon y Universidad de Leon. Cd-2. version 1.8, octubre de 2007. Junta de Castilla y Leon. Serie tecnica.

WHITTAKER, R.H. (1972). Evolution and measurement of species diversity. Taxon 21, 213-251.

WILLIAMS, C.; DAVIS, K. \& CHEYNE, P. (2003). El CBD para botánicos: una introducción al Convenio sobre la Diversidad Biológica para personas que trabajan con colecciones botánicas. Royal Botanical Garden, Kew. 